\title{
LACUNARY SERIES AND EXPONENTIAL MOMENTS
}

\author{
J. KUELBS ${ }^{1}$ AND W. A. WOYCZYNSKI ${ }^{2}$
}

\begin{abstract}
In the present paper we prove the boundedness of second exponential moments for the suprema of norms of partial sums of random series in Banach spaces, Sidon lacunary series on a compact Abelian group with coefficients in a Banach space, and for random lacunary series with similar coefficients. Exponential moments related to the law of iterated logarithm for lacunary series are also proved to be finite. Our results, even in case of standard trigonometric series with real coefficients, strengthen the classical theorem of Salem and Zygmund.
\end{abstract}

1. Introduction. Probabilistic independence and various group theoretic notions of lacunarity have often been interchangeable conditions in certain theorems in probability theory and harmonic analysis. A perfect example of this sort of phenomenon is Kolmogorov's law of the iterated logarithm for independent random variables and the law of the iterated logarithm for lacunary trigonometric series due to Salem and Zygmund [7] and [8]. There are many other such examples as well, and the point of this paper is to explore two new results involving the Rademacher functions $\left\{r_{j}(t): j\right\rangle$ 1\}-which are independent random variables-and their analogues for lacunary sums. Our results will generalize some theorems of Kahane [3], and will also specialize to give additional information for Hadamard lacunary trigonometric series in the setting of Salem and Zygmund.

2. Notation, definitions and preliminary lemmas. In what follows $B$ will denote a complex Banach space, $G$ a compact Abelian group, and $\left\{r_{j}(t)\right.$ : $j \geqslant 1\}$ the Rademacher functions on $[0,1]$. The characters on $G$ will be denoted by $\chi$ and the dual group itself by $X . d g$ stands for the normalized Haar measure on $G$.

The subset $\Delta \subseteq X$ is said to be a Sidon set if every bounded complex valued function on $\Delta$ is the restriction to $\Delta$ of the Fourier transform of a complex measure on $G$ with finite total variation.

Since $G$ is a compact Abelian group we know $X$ is a discrete locally compact Abelian group and further that $X$ has an abundance of Sidon sets.

Received by the editors March 28, 1977 and, in revised form, July 27, 1977.

AMS (MOS) subject classifications (1970). Primary 42A44, 60F15, 60G50; Secondary 60B99.

Key words and phrases. Lacunary series, exponential moments, law of the iterated logarithm, Sidon sets, Banach space valued random variables.

${ }^{1}$ Supported partially by NSF Grant MPS75-05855.

${ }^{2}$ Supported partially by NSF Grant MCS76-06935 while on leave from the Wrockaw University, Wrockaw, Poland, and the Institute of Mathematics, Polish Academy of Sciences. 
For example, each Hadamard lacunary sequence of exponential functions $\left\{\exp \left( \pm i n_{k} t\right): n_{k+1} / n_{k} \geqslant q>1\right\}$ is an example of a Sidon set when $G$ is the circle group and all dissociate sets in $G$ are Sidon (cf. [1, 442-445] and [6, 126-128]). For more facts on Sidon sets consult e.g. either [1] or [6].

Recall that the Banach space $B$ is said to be of Rademacher-type $p$, $1<p<2$, if $\exists A>0$ such that

$$
\int\left\|\sum r_{i}(t) x_{i}\right\|^{p} d t \leqslant A \sum\left\|x_{i}\right\|^{p}
$$

for any finite set $\left\{x_{j}\right\}$ on $B$. Banach spaces for which the modulus of smoothness $\rho(\tau)=O\left(\tau^{p}\right), \tau \rightarrow 0$, are of Rademacher-type $p$, and in particular, $L_{q}$ is of Rademacher-type $\min (2, p)$ whenever $q>p$. All $B$-spaces are of Rademacher type 1 . In the sequel $l_{p}(B)(1 \leqslant p<\infty)$ will denote the Banach space of all sequences $\left\{x_{j}\right\} \subseteq B$ such that

$$
\left\|\left\{x_{i}\right\}\right\|_{l^{P}(B)} \equiv\left(\sum\left\|x_{i}\right\|^{p}\right)^{1 / p}<\infty .
$$

$l_{\infty}(B)$ is defined in an analogous manner.

In the sequel we shall have need of the following lemmas.

LEMMA 2.1 [6, 121-122]. If $\Delta$ is a Sidon subset of $X$, then $\exists C>0$ such that every bounded complex function $f$ on $\Delta$ with $|f(x)|<1$ is the restriction to $\Delta$ of the Fourier transform of a complex measure $m$ on $G$ with $\|m\| \equiv|m|(G) \leqslant C$. Here $|m|$ is the total variation of $m$.

LEMMA 2.2. Let $\Phi(t)$ be a nonnegative, nondecreasing convex function on $[0, \infty)$. Let $\lambda_{1}, \lambda_{2}, \ldots$ be complex constants such that $\sup _{n}\left|\lambda_{n}\right| \leqslant \Gamma$. Let $X_{1}, X_{2}, \ldots$ be independent, symmetric $B$-valued random variables, and assume $\left\{a_{n}\right\}$ is a sequence of positive constants. Then, for any subset $\Lambda$ of the positive integers we have

$$
E\left\{\Phi \sup _{n \in \Lambda}\left\|\sum_{j=1}^{n} \lambda_{j} X_{j} / a_{n}\right\|\right\} \leqslant E\left\{\Phi\left(2 \Gamma \sup _{n \in \Lambda}\left\|\sum_{j=1}^{n} X_{j} / a_{n}\right\|\right)\right\} .
$$

Proof. See Lemma 5.7 of [2] for a method which handles this lemma immediately.

LEMMA 2.3. Let $X_{1}, X_{2}, \ldots$ be independent $B$-valued random variables such that $P\left(\left\|X_{j}\right\|<M_{j}\right)=1$ for certain $M_{j}<\infty(j \geqslant 1)$. If $S_{n}=X_{1}+\cdots+X_{n}$, then for all real $h$

$$
E\left(\exp \left(h\left\|S_{n}\right\|\right)\right)<\exp \left\{2 h^{2} \sum_{j=1}^{n} M_{j}^{2}+h E\left\|S_{n}\right\|\right\} .
$$

Proof. Define $E_{k} \eta \equiv E\left(\eta \mid X_{1}, \ldots, X_{k-1}\right), E_{n+1} \eta \equiv \eta, E_{1} \eta \equiv E \eta$, and set $\eta_{k}=E_{k+1}\left\|S_{n}\right\|-E_{k}\left\|S_{n}\right\|(k=1, \ldots, n)$. Then $\left\|S_{n}\right\|=\eta_{1}+\cdots+\eta_{n}+$ $E\left\|S_{n}\right\|$ so that 


$$
E\left(\exp \left(h\left\|S_{n}\right\|\right)\right)=E\left[\exp \left\{h E\left\|S_{n}\right\|+h \sum_{k=1}^{n-1} \eta_{k}\right\} E_{n}\left(\exp \left\{h \eta_{n}\right\}\right)\right) .
$$

Now $E_{k+1}\left\|S_{n}-X_{k}\right\|=E_{k}\left\|S_{n}-X_{k}\right\|$ by the independence of $X_{1}, \ldots, X_{n}$ and hence

$$
\eta_{k} \leqslant E_{k+1}\left\|S_{n}-X_{k}\right\|+E_{k+1}\left\|X_{k}\right\|-E_{k}\left\|S_{n}-X_{k}\right\|+E_{k}\left\|X_{k}\right\|=\left\|X_{k}\right\|+E\left\|X_{k}\right\|
$$

by the triangle inequality. Similarly $\eta_{k} \geqslant-\left\|X_{k}\right\|-E\left\|X_{k}\right\|$. Hence $\left|\eta_{k}\right| \leqslant$ $2 M_{k}$ and $E_{k} \eta_{k}=0$ so that in view of the inequality

$$
4 M_{k} \exp \left(h \eta_{k}\right) \leqslant\left(2 M_{k}-\eta_{k}\right) e^{-2 h M_{k}}+\left(\eta_{k}-\left(-2 M_{k}\right)\right) e^{2 h M_{k}},
$$

(implied by convexity of $\exp (h u)$ ) we have

$$
E_{k}\left(\exp \left(h \eta_{k}\right)\right) \leqslant \cos h 2 h M_{k} \leqslant \exp \left\{2 h^{2} M_{k}^{2}\right\}
$$

since $\left(e^{x}+e^{-x}\right) \leqslant 2 e^{x^{2} / 2}$ for all $x$. Using (2.4) in (2.3) we obtain

$$
E\left(\exp \left(h\left\|S_{n}\right\|\right)\right) \leqslant \dot{E}\left[\exp \left\{h \sum_{k=1}^{n-1} \eta_{k}\right\}\right) \exp \left\{h E\left\|S_{n}\right\|+2 h^{2} M_{n}^{2}\right\} .
$$

Iterating $(n-1)$ more times we thus have $(2.2)$ so the lemma is proved.

3. Exponential moments of sums of $B$-valued random variables. The following general result for the Rademacher functions was originally proved for real Banach spaces, but its generalization to complex Banach spaces is straightforward.

THEOREM 3.1 [5]. If $\left\{x_{j}: j \geqslant 1\right\}$ is a fixed sequence in $B$ such that $\sum r_{j}(t) x_{j}$ converges a.s. $(t)$ in $B$, then for all $\beta>0$

$$
\int_{0}^{1} \exp \left\{\beta \sup _{n}\left\|\sum_{j=1}^{n} r_{j}(t) x_{j}\right\|^{2}\right\} d t<\infty
$$

Another result involving exponential moments of random vector series is the following.

THEOREM 3.2. Assume $\left\{X_{j}: j \geqslant 1\right\}$ is a sequence of random variables on $(\Omega, \mathcal{F}, P)$ with values in the Banach space $B$ of Rademacher-type $p$, and independent of the Rademacher functions $\left\{r_{j}(t): j \geqslant 1\right\}$. If there exists a bounded set $V \subseteq l_{p}(B)$ such that $P\left(\omega:\left\{X_{j}(\omega): j \geqslant 1\right\} \subseteq V\right)=1$, then there exists $\beta_{0}>0$ such that for $\beta \leqslant \beta_{0}$ we have

$$
\underset{\omega \in \Omega}{\operatorname{ess} \sup } \int_{0}^{1} \exp \left\{\beta \sup \left\|\sum_{j=1}^{n} r_{j}(t) X_{j}(\omega)\right\|^{2}\right\} d t<\infty
$$

Proof. First observe that, by Lemma 2.3 , for all $\omega \in \Omega$ 


$$
\begin{array}{r}
P\left\{t:\left\|\sum_{j=1}^{n} r_{j}(t) X_{j}(\omega)\right\|>\lambda\right] \leqslant e^{-\alpha \lambda} \int_{0}^{1} \exp \left\{\alpha\left\|\sum_{j=1}^{n} r_{j}(t) X_{j}(\omega)\right\|\right\} d t \\
\leqslant \exp \left\{2 \alpha^{2} \sum_{j=1}^{n}\left\|X_{j}(\omega)\right\|^{2}-\alpha\left[\lambda-\int_{0}^{1}\left\|\sum_{j=1}^{n} r_{j}(t) X_{j}(\omega)\right\| d t\right\}\right. \\
\leqslant \exp \left\{-\left[\lambda-\int_{0}^{1}\left\|\sum_{j=1}^{n} r_{j}(t) X_{j}(\omega)\right\| d t\right]^{2} / 8 \sum_{j=1}^{n}\left\|X_{j}(\omega)\right\|^{2}\right\}
\end{array}
$$

by minimizing with respect to $\alpha$. Now, because $B$ is of Rademacher-type $p$ we have

$$
\Lambda \equiv \underset{\omega \in \Omega}{\operatorname{ess} \sup \sup } \int_{0}^{1}\left\|\sum_{j=1}^{n} r_{j}(t) X_{j}(\omega)\right\| d t<\infty
$$

so that for $\lambda \geqslant 2 \Lambda$ and $M$ such that $\Sigma\left\|x_{j}\right\|^{2} \leqslant M$ for each $\left\{x_{j}\right\} \in V$ we have for almost all $\omega$ that

$$
P\left[t:\left\|\sum_{j=1}^{n} r_{j}(t) X_{j}(\omega)\right\|>\lambda\right] \leqslant \exp \left\{-\lambda^{2} / 32 M\right\} .
$$

By Lévy's maximal inequality we therefore have for almost all $\omega$ that $\lambda \geqslant 2 \Lambda$ implies

$$
P\left[t: \sup _{n}\left\|\sum_{j=1}^{n} r_{j}(t) X_{j}(\omega)\right\|>\lambda\right] \leqslant 2 \exp \left\{-\lambda^{2} / 32 M\right\} .
$$

Thus (3.2) holds since for almost all $\omega$ the above inequality implies for $\beta \leqslant 1 / 32 M$ that

$$
\int_{0}^{1} \exp \left\{\beta \sup _{n}\left\|\sum_{j=1}^{n} r_{j}(t) X_{j}(\omega)\right\|^{2}\right\} d t<\infty .
$$

REMARK 3.1. From the above proof it is clear that in Theorem 3.2 the assumption that $B$ is of Rademacher-type $p$ may be replaced by condition (3.3).

COROLlary 3.1. Assume the hypotheses in Theorem 3.2 and that

$$
\lim _{N} \sup _{\left\{x_{j}\right\} \in V} \sum_{j=N}^{\infty}\left\|x_{j}\right\|^{p}=0 .
$$

Then (3.2) holds for all $\beta>0$.

Proof. Fix $\beta>0$ and choose $N$ such that

$$
\sup _{\left\{x_{j}\right\} \in V} \sum_{j=N}^{\infty}\left\|x_{j}\right\|^{p} \leqslant \varepsilon
$$


where $\varepsilon>0$ is such that $\beta<1 / 32 \varepsilon$. Then by the argument given in the proof of Theorem 3.2 we have

$$
\underset{\omega \in \Omega}{\operatorname{ess} \sup } \int_{0}^{1} \exp \left\{\beta \sup _{n>N}\left\|\sum_{j=N}^{n} r_{j}(t) X_{j}(\omega)\right\|^{2}\right\} d t<\infty
$$

so that the corollary is proved.

Now we turn to the investigation of exponential moments related to the law of the iterated logarithm. $L(x)$ will denote the function which is 1 for $x \leqslant e$ and $\log x$ elsewhere, and $a_{n}$ will stand for $\left(2 \sigma_{n}^{2} L L \sigma_{n}^{2}\right)^{1 / 2}$.

THeOREM 3.3 [4]. If $\left\{x_{j}: j \geqslant 1\right\}$ is a bounded sequence in $B$ and $\left\{c_{j}\right\} a$ sequence of nonnegative constants such that

(i) $\sigma_{n}^{2} \equiv c_{1}^{2}+\cdots+c_{n}^{2} \rightarrow \infty$ as $n \rightarrow \infty$,

(ii) $c_{n}^{2} / \sigma_{n}^{2} \rightarrow 0$ as $n \rightarrow \infty$, and

(iii) $\left\{\sum_{j=1}^{n} c_{j} r_{j}(t) x_{j} / \sigma_{n}: n \geqslant 1\right\}$ is bounded in probability, then for all $\beta>0$

$$
\int_{0}^{1} \exp \left\{\beta \sup _{n}\left\|\sum_{j=1}^{n} c_{j} r_{j}(t) x_{j} / a_{n}\right\|^{2}\right\} d t<\infty .
$$

REMARK 3.2. If $B$ is of Rademacher-type 2, then condition (iii) is automatically satisfied.

One can also obtain the following "log $\log$ " analogue of Theorem 3.2.

THEOREM 3.4. Assume $\left\{X_{j}: j \geqslant 1\right\}$ is a sequence of random variables on $(\Omega, \mathcal{F}, P)$ with values in a Banach space $B$ of Rademacher-type 2, and, independent of the Rademacher functions $\left\{r_{j}(t), j \geqslant 1\right\}$. Let $\left\{c_{j}\right\}$ be a sequence of nonnegative constants satisfying (i), (ii) of Theorem 3.3. If there exists a bounded $V \subseteq l_{\infty}(B)$ such that $P\left(\omega:\left\{X_{j}(\omega): j \geqslant 1\right\} \subseteq V\right)=1$ then for all $\beta>0$ we have

$$
\underset{\omega \in \Omega}{\operatorname{ess} \sup } \int_{0}^{1} \exp \left\{\beta \sup _{n}\left\|\sum_{j=1}^{n} c_{j} r_{j}(t) X_{j}(\omega) / a_{n}\right\|^{2}\right\} d t<\infty .
$$

Proof. By assumption

$$
M \equiv \underset{\omega \in \Omega}{\operatorname{ess} \sup \sup }\left\|X_{j}(\omega)\right\|^{2}<\infty
$$

and

$$
\begin{aligned}
\int_{0}^{1}\left\|\sum_{j=1}^{n} c_{j} r_{j}(t) X_{j}(\omega) / \sigma_{n}\right\| d t & \leqslant\left(A \sigma_{n}^{-2} \sum_{j=1}^{n} c_{j}^{2}\left\|X_{j}(\omega)\right\|^{2}\right)^{1 / 2} \\
& \leqslant A^{1 / 2} M^{1 / 2}
\end{aligned}
$$

for almost all $\omega$ because $B$ is of Rademacher-type 2 . Hence 


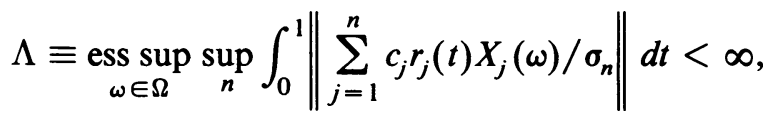

and proceeding as in the proof of Theorem 3.2, we have for all $\omega, \eta$ and $\lambda \geqslant 2 \Lambda$

$$
\begin{aligned}
& P\left\{t:\left\|\sum_{j=1}^{n} c_{j} r_{j}(t) X_{j}(\omega) / \sigma_{n}\right\| \lambda\right] \\
& \leqslant \exp \left\{-\left(\lambda-\int_{0}^{1}\left\|\sum_{j=1}^{n} c_{j} r_{j}(t) X_{j}(\omega) / \sigma_{n}\right\| d t\right)^{2}\right. \\
& \left.\qquad\left[8 \sum_{j=1}^{n} \frac{c_{j}^{2}\left\|X_{j}(\omega)\right\|^{2}}{\sigma_{n}^{2}}\right]^{-1}\right\} \\
& \leqslant \exp \left\{-\lambda^{2} / 32 M\right\} .
\end{aligned}
$$

Define for $r=1,2, \ldots$

$$
M_{r}=\sup _{n \geqslant r}\left\|\sum_{j=1}^{n} c_{j} r_{j}(t) X_{j}(\omega) / a_{n}\right\|
$$

and set $n_{k}=\min \left\{j: \sigma_{j}^{2} \geqslant 2^{k}\right\} \quad(k \geqslant 2)$ with $n_{1}=1$. Further, put $k(r)=$ $\max \left\{j: n_{j} \leqslant r\right\}$ for $r \geqslant 1$ and define

$$
R_{n}(t, \omega)=\sum_{j=1}^{n} c_{j} r_{j}(t) X_{j}(\omega) \quad(n \geqslant 1) .
$$

Then by Lévy's maximal inequality and (3.6) we have for almost all $\omega \in \Omega$ that

$$
\begin{aligned}
P\left(t: M_{r} \geqslant \lambda\right) \leqslant & \sum_{k>k(r)+1} P\left(t: \max _{n_{k-1}<n<n_{k}}\left\|R_{n} / a_{n}\right\| \geqslant \lambda\right) \\
& \leqslant \sum_{k>k(r)+1} 2 P\left(t:\left\|R_{n_{k}} / a_{n_{k-1}}\right\| \geqslant \lambda\right) \\
& =2 \sum_{k>k(r)+1} P\left(t:\left\|R_{n_{k}}\right\| / \sigma_{n_{k}} \geqslant \lambda\left(\sigma_{n_{k-1}} / \sigma_{n_{k}}\right) \sqrt{2 L L \sigma_{n_{k-1}}^{2}}\right) \\
& =2 \sum_{k>k(r)+1} P\left(t:\left\|R_{n_{k}}\right\| / \sigma_{n_{k}} \geqslant(\lambda / 4) \sqrt{2 L L 2^{n-1}}\right) \\
& \leqslant 2 \sum_{k>k(r)+1} \exp \left\{-\lambda^{2} L L 2^{k-1} / 256 M\right\}
\end{aligned}
$$

provided $r$ is sufficiently large so that $k \geqslant k(r)+1$ implies $\sigma_{n_{k-1}} / \sigma_{n_{k}} \geqslant 1 / 4$. Such an $r$ exists since (i) and (ii) of Theorem 3.3 imply that $\sigma_{n_{k-1}} / \sigma_{n_{k}} \rightarrow 1 / 2$ as $k \rightarrow \infty$.

Now (3.4) holds if we show that for each $\beta>0$ there exists an $r$ such that 


$$
\underset{\omega \in \Omega}{\operatorname{ess} \sup } \int_{0}^{1} \exp \left\{\beta M_{r}^{2}(t, \omega)\right\} d t<\infty .
$$

To verify (3.8) choose $\alpha$ so that $\log \alpha>\beta \max \left(64 \Lambda^{2}, 512 M, 2\right)$, and $r$ such that $k \geqslant k(r)+1$ implies $\sigma_{n_{k-1}} / \sigma_{n_{k}} \geqslant 1 / 4$ (and hence (3.7) holds) and $\left(L L 2^{k-1} / 256 M\right)-\beta \geqslant \beta$. Then by (3.7) we have for almost all $\omega \in \Omega$ that

$$
\begin{aligned}
& \int_{0}^{1} \exp \left\{\beta M_{r}^{2}(t, \omega)\right\} d t=\int_{0}^{\infty} P\left(t: \exp \left\{\beta M_{r}^{2}(t, \omega)\right\}>s\right) d s \\
& \leqslant \alpha+\int_{\alpha}^{\infty} P\left(t: M_{r}(t, \omega)>(\log s / \beta)^{1 / 2}\right) d s \\
& \quad=\alpha+\int_{(\log \alpha / \beta)^{1 / 2}}^{\infty} P\left(t: M_{r}(t, \omega)>u\right) 2 \beta u e^{\beta u^{2}} d u \\
& \leqslant \alpha+2 \sum_{k>k(r)+1} \int_{(\log \alpha / \beta)^{1 / 2}}^{\infty} \exp \left\{-u^{2}\left[\left(L L 2^{k-1} / 256 M\right)-\beta\right]\right\} 2 \beta u d u \\
& \leqslant \alpha+2 \sum_{k>k(r)+1} \exp \left\{-\Lambda_{k} \log \alpha\right\} / \Lambda_{k}
\end{aligned}
$$

(where $\left.\Lambda_{k}=\beta^{-1}\left[\left(L L 2^{k-1} / 256 M\right)-\beta\right] \geqslant 1\right)$

$$
\leqslant \alpha+2 \alpha \sum_{k>k(r)+1}((k-1) L 2)^{-\gamma}<\infty,
$$

since $\gamma=(\log \alpha) / \beta 256 M \geqslant 2$. Since the above estimates are uniform on almost all $\omega \in \Omega$ we have (3.8) and hence the theorem is proved.

REMARK 3.3. From the above proof it is clear that in Theorem 3.4 the assumption that $B$ is of Rademacher-type 2 may be replaced by condition (3.5).

4. Exponential moments of lacunary and random lacunary series. The following proposition is fundamental in what follows and relates exponential moments of Rademacher series to exponential moments of lacunary series.

Proposition 4.1. Let $\Delta=\left\{\chi_{j}: j \geqslant 1\right\} \subseteq X$ be a Sidon set, $C$ the constant given by Lemma 2.1, $\left\{x_{j}: j \geqslant 1\right\}$ a sequence in $B$ and $\left\{\alpha_{j}: j \geqslant 1\right\}$ a bounded sequence of complex numbers with $\Gamma=\sup _{j}\left|\alpha_{j}\right|$. Then for any sequence $a_{n}$ of positive numbers

$$
\begin{aligned}
\left.\int_{G} \exp _{n} \sup _{n}\left\|\sum_{j=1}^{n} \alpha_{j} x_{j}(g) x_{j} / a_{n}\right\|^{2}\right\} d g \\
\leqslant \int_{0}^{1} \exp \left\{2 C^{2} \Gamma \sup _{n}\left\|\sum_{j=1}^{n} r_{j}(t) x_{j} / a_{n}\right\|^{2}\right\} d t .
\end{aligned}
$$

Proof. Define the "randomization" of the trigonometric polynomial

$$
S_{n}(g)=\sum_{j=1}^{n} \alpha_{j} \chi_{j}(g) x_{j} / a_{n}
$$


as follows

$$
T_{n}(g, t)=\sum_{j=1}^{n} \alpha_{j} \chi_{j}(g) r_{j}(t) x_{j} / a_{n} \quad(n \geqslant 1) .
$$

Now we apply Lemma 2.1 to produce for each $t(0 \leqslant t \leqslant 1)$ complex measure $m_{t}$ on $G$ such that the Fourier transform of $m_{t}$ satisfies

$$
\int_{G} \chi_{j}(g) m_{t}(d g)=r_{j}(t) \quad(j \geqslant 1)
$$

and $C \equiv \sup \left|m_{t}\right|(G)<\infty$. Hence for each $t(0 \leqslant t \leqslant 1)$ we have

$$
\begin{aligned}
\int_{G} T_{n}(g+h, t) m_{t}(d h) & =\sum_{j=1}^{n} \alpha_{j} r_{j}(t) x_{j} \int_{G} \chi_{j}(g) \chi_{j}(h) m_{t}(d h) / a_{n} \\
& =\sum_{j=1}^{n} \alpha_{j} \chi_{j}(g) x_{j} r_{j}^{2}(t) / a_{n}=S_{n}(g),
\end{aligned}
$$

and we easily obtain from Jensen's inequality that

$$
\begin{aligned}
\sup _{n<N}\left\|S_{n}(g)\right\|^{2} & =\sup _{n<N}\left\|\int_{G} T_{n}(g+h, t) m_{t}(d h)\right\|^{2} \\
& \leqslant\left(\int_{G} \sup _{n<N}\left\|T_{n}(g+h, t)\right\|\left|m_{t}\right|(d h)\right)^{2} \\
& \leqslant C \int_{G} \sup _{n<N}\left\|T_{n}(g+h, t)\right\|^{2}\left|m_{t}\right|(d h) .
\end{aligned}
$$

Applying Jensen's inequality again we have that

$$
\begin{gathered}
\exp \left\{\sup _{n<N}\left\|S_{n}(g)\right\|^{2}\right\} \leqslant \exp \left\{C \int_{G} \sup _{n<N}\left\|T_{n}(g+h, t)\right\|^{2}\left|m_{t}\right|(d h)\right\} \\
\leqslant \int_{G} \exp \left\{C^{2} \sup _{n<N}\left\|T_{n}(g+h, t)\right\|^{2}\right\}\left\|m_{t}\right\|^{-1}\left|m_{t}\right|(d h) .
\end{gathered}
$$

Integrating both sides with respect to $d g$ we have by the translation invariance of Haar measure that

$$
\begin{aligned}
\int_{G} \exp & \left\{\sup _{n<N}\left\|S_{n}(g)\right\|^{2}\right\} d g \\
& \leqslant \int_{G}\left[\int_{G} \exp \left\{C^{2} \sup _{n<N}\left\|T_{n}(g+h, t)\right\|^{2}\right\} d g\right]\left\|m_{t}\right\|^{-1}\left|m_{t}\right|(d h) \\
& =\int_{G}\left[\int_{G} \exp \left\{C^{2} \sup _{n<N}\left\|T_{n}(g, t)\right\|^{2}\right\} d g\right]\left\|m_{t}\right\|^{-1}\left|m_{t}\right|(d h) \\
& =\int_{G} \exp \left\{C^{2} \sup _{n<N}\left\|T_{n}(g, t)\right\|^{2}\right\} d g .
\end{aligned}
$$

Integrating both sides with respect to the Lebesgue measure we get, by Fubini's theorem, that 


$$
\begin{aligned}
& \int_{G} \exp \left\{\sup _{n<N}\left\|S_{n}(g)\right\|^{2}\right\} d g \\
& \leqslant \int_{G}\left\{\int_{0}^{1} \exp \left\{C^{2} \sup _{n<N}\left\|\sum_{j=1}^{n} \alpha_{j} x_{j}(g) x_{j} r_{j}(t) / a_{n}\right\|^{2}\right\} d t\right] d g \\
& \quad \leqslant \int_{G} \exp \left\{2 \Gamma C^{2} \sup _{n<N}\left\|\sum_{j=1}^{n} r_{j}(t) x_{j} / a_{n}\right\|^{2}\right\} d t
\end{aligned}
$$

where the last inequality results from Lemma 2.2 . Thus the theorem is proved.

The following theorem is an immediate corollary to Theorem 3.1 and Proposition 4.1.

TheOREM 4.1. Let $\Delta=\left\{\chi_{j}: j \geqslant 1\right\} \subseteq X$ be a Sidon set and assume $\left\{x_{j}\right.$ : $j \geqslant 1\}$ is a fixed subsequence of $B$ such that $\sum r_{j}(t) x_{j}$ converges in $B(d t)$-almost surely. Then for any bounded sequence of complex numbers $\left(\alpha_{j}\right)$ we have that

$$
\int_{G} \exp \left\{\sup _{n}\left\|\sum_{j=1}^{n} \alpha_{j} x_{j}(g) x_{j}\right\|^{2}\right\} d g<\infty .
$$

In case $G$ is the unit circle we have the following corollary which strengthens the result in $[9$, p. 215].

COROLlaRY 4.1. Let $\left\{x_{j}: j \geqslant 1\right\}$ be a fixed sequence in $B$ such that $\sum r_{j}(t) x_{j}$ converges in $B$ dt-almost surely. If $\left\{n_{j}: j \geqslant 1\right\}$ is a sequence of positive integers which is a Sidon set, and $\left\{a_{j}\right\},\left\{b_{j}\right\}$ are bounded sequences of complex numbers then

$$
\int_{0}^{2 \pi} \exp \left\{\sup _{n}\left\|\sum_{j=1}^{n}\left[a_{j} \cos n_{j} s+b_{j} \sin n_{j} s\right] x_{j}\right\|^{2}\right\} d s<\infty .
$$

REMARK 4.1. In case $B$ is of Rademacher-type $p, 1<p \leqslant 2$, then the assumption in Theorem 4.1 and Corollary 4.1 that $\sum r_{j}(t) x_{j}$ converges a.s. may be replaced by the condition $\Sigma\left\|x_{j}\right\|^{p}<\infty$.

Concerning random lacunary series we have the following straightforward corollary to Theorem 3.2 and Proposition 4.1.

TheOrem 4.2. Let. $\Delta=\left\{\chi_{j}: j \geqslant 1\right\} \subset X$ be a Sidon set and assume $\left\{X_{j}\right.$ : $j \geqslant 1\}$ is a sequence of random variables on $(\Omega, \mathcal{F}, P)$ with values in the Banach space $B$ of Rademacher-type $p$. If there exists a bounded $V \subseteq l_{p}(B)$ such that $P\left(\omega:\left\{X_{j}(\omega): j \geqslant 1\right\} \subset V\right)=1$ then there exists $\beta_{0}>0$ such that for $\beta \leqslant \beta_{0}$ we have

$$
\underset{\omega \in \Omega}{\operatorname{ess} \sup } \int_{G} \exp \left\{\beta \sup _{n}\left\|\sum_{j=1}^{n} x_{j}(g) X_{j}(\omega)\right\|^{2}\right\} d g<\infty .
$$

REMARK 4.2. In analogy to Corollary 3.1 if in Theorem 4.2 


$$
\lim _{N} \sup _{\left\{x_{j}\right\} \in V} \sum_{j=N}^{\infty}\left\|x_{j}\right\|^{p}=0
$$

then (4.2) holds for all $\beta>0$.

As an immediate corollary to Theorem 3.3 and Proposition 4.1 (with $x_{j}$ replaced by $c_{j} x_{j}$ ) we have the following theorem on exponential moments related to the law of iterated logarithm for lacunary series. Below $a_{n}=$ $\left(2 \sigma_{n}^{2} L L \sigma_{n}^{2}\right)^{1 / 2}$.

THEOREM 4.3. Let $\Delta=\left\{\chi_{j}: j \geqslant 1\right\} \subseteq X$ be a Sidon set and assume $\left\{x_{j}\right\}$ is a fixed sequence in $B$ and $\left\{c_{j}\right\}$ is a sequence of nonnegative numbers such that (i)-(iii) of Theorem 3.3 are satisfied. Then for all bounded complex sequences $\left\{\alpha_{j}\right\}$ we have

$$
\int_{G} \exp \left\{\beta \sup _{n}\left\|\sum_{j=1}^{n} \alpha_{j} c_{j} x_{j}(g) x_{j} / a_{n}\right\|^{2}\right\} d g<\infty .
$$

The next result gives us additional information beyond that given in the classical result of Salem and Zygmund [7]. Furthermore, the Remark 3.2 amplifies the relationship between this result and [7].

COROLlary 4.2. Let $\left\{x_{j}: j \geqslant 1\right\} \subset B$ and let $\left\{c_{j}\right\}$ be a sequence of nonnegative constants such that (i)-(iii) of Theorem 3.3 hold. If $\left\{n_{j}: j \geqslant 1\right\}$ is a sequence of positive integers which is a Sidon set, and $\left\{\alpha_{j}\right\}$ and $\left\{\beta_{j}\right\}$ are complex numbers such that $c_{j}^{2}=\left|\alpha_{j}\right|^{2}+\left|\beta_{j}\right|^{2}(j \geqslant 1)$ then for all $\beta>0$

$$
\int_{0}^{2 \pi} \exp \left\{\beta \sup _{n}\left\|\sum_{j=1}^{n}\left[\alpha_{j} \cos n_{j} s+\beta_{j} \sin n_{j} s\right] x_{j} / a_{n}\right\|^{2}\right\} d s<\infty .
$$

Our last result is a "log log" version of Theorem 4.2 and is an immediate corollary to Theorem 3.4 and Proposition 4.1.

Theorem 4.4. Let $\Delta=\left\{\chi_{j}: j \geqslant 1\right\} \subset X$ be a Sidon set and assume that $\left\{X_{j}\right\}$ is a sequence of random variables on $(\Omega, \mathscr{F}, P)$ with values in the Banach space $B$ of Rademacher-type 2. Assume $\left\{X_{j}\right\}$ is independent of the Rademacher functions $\left\{r_{j}(t): j \geqslant 1\right\}$, and that $\left\{c_{j}\right\}$ satisfies (i)-(ii) of Theorem 3.3. If there exists a bounded $V \subseteq l_{\infty}(B)$ such that $P\left(\omega:\left\{X_{j}(\omega): j \geqslant 1\right\} \subseteq V\right)=1$ then for all $\beta>0$ we have

$$
\underset{\omega \in \Omega}{\operatorname{ess} \sup } \int_{G} \exp \left\{\beta \sup _{n}\left\|\sum_{j=1}^{n} c_{j} \chi_{j}(g) X_{j}(\omega) / a_{n}\right\|^{2}\right\} d g<\infty
$$

\section{REFERENCES}

1. E. Hewitt and K. A. Ross, Abstract harmonic analysis. II, Springer-Verlag, Berlin and New York, 1967.

2. N. C. Jain and M. B. Marcus, Integrability of infinite sums of independent vector valued random variables, Trans. Amer. Math. Soc. 212 (1975), 1-36.

3. J. P. Kahane, Some random series of functions, Heath, Lexington, Mass., 1968.

4. J. Kuelbs, Some exponential moments of sums of independent random variables, Trans. Amer. Math. Soc. (to appear). 
5. S. Kwapien, $A$ theorem on the Rademacher series with vector valued coefficients, Probability in Banach Spaces, Lecture Notes in Math., vol. 526, Springer-Verlag, Berlin and New York, 1976, pp. 157-158.

6. W. Rudin, Fourier analysis on groups, Interscience, New York, 1962.

7. R. Salem and A. Zygmund, La loi du logarithme itéré pour les séries trigonométriques lacunaires, Bull. Sci. Math. 74 (1950), 209-224.

8. M. Weiss, The law of the iterated logarithm for lacunary trigonometric series, Trans. Amer. Math. Soc. 91 (1959), $444-469$.

9. A. Zygmund, Trigonometric series. I, Cambridge Univ. Press, London, 1959.

Department of Mathematics, University of Wisconsin, Madison, Wisconsin 53706

DePartment of Mathematics, NorThWESTERn University, Evanston, IllinOIS 60201 